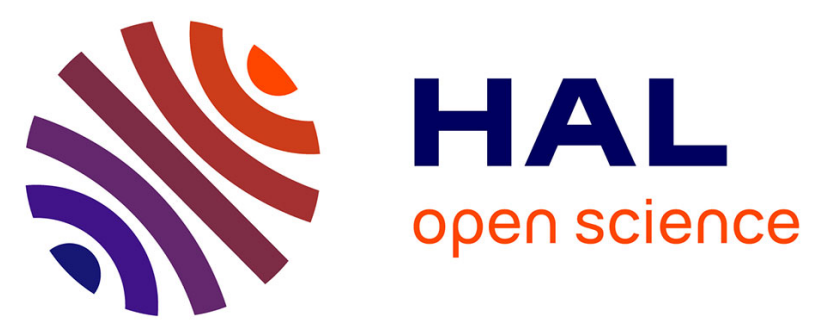

\title{
Monte Carlo simulation of radionuclide migration in fractured rock for the performance assessment of radioactive waste repositories
}

\author{
F. Cadini, J. de Sanctis, I. Bertoli, Enrico Zio
}

\section{- To cite this version:}

F. Cadini, J. de Sanctis, I. Bertoli, Enrico Zio. Monte Carlo simulation of radionuclide migration in fractured rock for the performance assessment of radioactive waste repositories. Reliability Engineering and System Safety, 2013, 111, pp.241-247. 10.1016/j.ress.2012.10.002 . hal-00771552

\section{HAL Id: hal-00771552}

https://hal-centralesupelec.archives-ouvertes.fr/hal-00771552

Submitted on 8 Jan 2013

HAL is a multi-disciplinary open access archive for the deposit and dissemination of scientific research documents, whether they are published or not. The documents may come from teaching and research institutions in France or abroad, or from public or private research centers.
L'archive ouverte pluridisciplinaire HAL, est destinée au dépôt et à la diffusion de documents scientifiques de niveau recherche, publiés ou non, émanant des établissements d'enseignement et de recherche français ou étrangers, des laboratoires publics ou privés. 


\title{
MONTE CARLO SIMULATION OF RADIONUCLIDE MIGRATION IN FRACTURED ROCK FOR THE PERFORMANCE ASSESSMENT OF RADIOACTIVE WASTE REPOSITORIES
}

\author{
F. Cadini ${ }^{1}$, J. De Sanctis ${ }^{1}$, I. Bertoli ${ }^{1}$, E. Zio ${ }^{1,2}$ \\ ${ }^{1}$ Dipartimento di Energia - Politecnico di Milano \\ Via Ponzio 34/3, I-20133 Milan, Italy \\ francesco.cadini@polimi.it \\ ${ }^{2}$ Chair on Systems Science and the Energetic challenge, \\ European Foundation for New Energy- Électricité de France \\ Ecole Centrale Paris and Supelec
}

\begin{abstract}
The estimation of radionuclide migration within a fractured medium is a fundamental task in any performance assessment aimed at verifying the protection offered by radioactive waste repositories. In this paper, we present a novel application of a dualpermeability, Monte Carlo simulation approach based on Kolmogorov-Dmitriev (KD) theory of branching stochastic processes. The Monte Carlo simulation scheme allows to easily handle the high degree of complexity of the problem, accounting for nonhomogeneities both in space and time. Particular attention is here devoted to the inclusion of time-dependencies. An illustration is given with reference to a realistic case study of ${ }^{239} \mathrm{Pu}$ migration.
\end{abstract}

\section{INTRODUCTION}

Fractures are the main responsible of contaminant transport in low-permeability formations. The water flow and the associated advective transport in the host rock are negligible with respect to what is occurring in the preferential pathways offered by the fractures, in particular if interconnected [1][2]. At the same time, the attenuation mechanism of molecular diffusion into the solid matrix can constitute a significant retardation mechanism [3]. For these reasons, the quantitative analysis of the processes of contaminant migration through fractured media plays a fundamental role in safety analyses, e.g. for the assessment of land disposal sites and deep disposal 
wells for chemical or low-level radioactive wastes, or the Performance Assessment (PA) of geological repositories for high-level nuclear wastes [4][5]. An example of the latter is provided by the PA for the Waste Isolation Pilot Plant (WIPP), where the analysis for radionuclide transport in the Culebra Dolomite involved movement within and exchange between fractures and a surrounding matrix [4]. Another example is provided by the 2008 PA for the proposed repository for radioactive waste at Yucca Mountain (YM), Nevada; in this analysis, fracture flow and associated radionuclide transport were modeled in both the unsaturated zone (UZ) surrounding the waste disposal drifts [6][7][8] and in the saturated zone (SZ) located below the UZ [9][10][11].

Many approaches have been proposed in the literature to tackle this problem. Within the simplifying setting of a single, longitudinal fracture, both analytical [12][13] and numerical [14] solutions have been successfully compared to the experimental results of [15]. The interested reader may also refer to [16] for a review of the most important existing approaches. However, when dealing with more complex domains, characterized by large-scale fracture networks, detailed analytical approaches become impractical and the extension of the local-scale numerical schemes to the whole migration domain is prohibitive. In these cases, dual- or, more generally, multiplecontinua approaches offer a viable alternative based on sets of coupled classical advectiondispersion equations: here, the detailed physical and geometrical information on the fractured migration domain is condensed in lumped, effective parameters thus allowing in principle for analytical or numerical solutions.

In this paper, we exploit a Monte Carlo simulation scheme based on the Kolmogorov-Dmitriev (KD) theory of branching stochastic processes [16][17][18] for the solution of a dual-permeability migration problem within a realistic performance assessment case study. This numerical approach has been chosen because it allows a straightforward inclusion of i) many physical and chemical processes by simply introducing new particle types (see [16] for some examples); ii) complex geometries of the migration domain and iii) inhomogeneities both in time and space. In particular, in a realistic setting, the geometric and hydrologic properties of the transport media may be subject to changes which can be both continuous and discrete in time. For example, such modifications may include the closure of fractures in salt deposits due to the plasticity of the medium [19][20], the decrease in fracture aperture due to slow deposition [21], seasonal water content changes in seasons due to rainfalls [22], thermo-hydraulic-mechanical-chemical (THMC) couplings which can affect the permeability of the media and the average hydraulic gradient [23][24][25][26] or the abrupt changes in the fracture aperture due to shock events (e.g. earthquakes, volcanic eruptions, etc.) [27]. A significant, practical example of the importance of accounting for such evolving system properties is provided by the PA of the WIPP mentioned above, where the evolution of the 
repository system due to brine inflow, gas generation, system pressurization, and pressure-induced fracturing was analyzed [4][5][28].

The flexibility of the proposed modeling approach easily allows dealing with the time-dependencies introduced by these phenomena. In fact, in the following, this is demonstrated through the application to a realistic case study of performance assessment of a waste repository located in a highly fractured zone [29] subject to seismic activity.

The paper is organized as follows. In Section 2, the principal features of the dual-permeability, Monte Carlo simulation-based model are recalled. Section 3, describes the application of the model to a case study of literature [29]. Conclusions on the capabilities of the proposed procedure are drawn in Section 4.

\section{THE MODEL}

In this Section, we consider the one-dimensional, Monte Carlo simulation, dual-permeability scheme introduced in [17] and based on the Kolmogorov-Dmitriev (KD) theory of branching stochastic processes [16][18].

The key feature of KD modeling is that different types of particles, characterized by different stochastic behaviors, are introduced to represent the solute in its possible different states (physical or chemical) and regions of space (positions). Partial differential equations, known as the Forward Kolmogorov Equations (FKEs), are then derived for the expected values of the different particles' populations, which are proportional to the solute concentrations. Within a Markovian description of the stochastic space-time evolution of a system of different particles, the KD model is naturally suited to a Monte Carlo particle tracking-based solution of the associated FKEs: from the probability density functions of the model, a large number of realizations of the migration fates of contaminant particles are simulated.

For simplicity, in what follows we shall refer to a one-dimensional domain [16][17], which is subdivided in $N_{z}$ discrete zones, $z=1,2, \ldots, N_{z}$. The objective is that of determining the amount of contaminant present at each time in each zone of the porous matrix and system of fractures. Two categories of particles are introduced: the solutons, which are the particles of contaminant migrating in the porous matrix, and the fracturons, which are the particles of contaminant migrating in the fractures. Thus, the system is made up of $m=2 N_{z}$ different kinds of particles, i.e. the solutons and the fracturons in the zones $z=1,2, \ldots, N_{z}$. Each particle may disappear or give rise to one particle of the $m-1$ remaining kinds according to given transition probability laws. It is assumed that: i) the 
stochastic process is Markovian, i.e. a particle of the $k^{\text {th }}$ kind, $k=1, \ldots, m$, gives rise to a branching process independently of its past history; ii) the process is linear, i.e. the particles do not interact among each other; iii) within a generic time interval $(t, t+d t)$, with $d t$ sufficiently small, only one particle transition may occur.

Within this framework, in the time interval $d t$ a soluton can undergo an exchange transition in zone $z$, thus transforming into a fracturon in zone $z$; the probability of occurrence of this transition, conditioned on the fact that the particle was a soluton at time $t$ in zone $z$, is $l_{s \rightarrow f, z}(t) d t$, where $l_{s \rightarrow f, z}(t)$ is the corresponding transition rate. Alternatively, within the same time interval $d t$, the soluton can travel to one of the neighboring zones, $z+1$ or $z-1$, with transition rates $f_{s}(t)$ (forward) and $b_{s}(t)$ (backward), respectively. Because of the continuity of the underlying physical phenomenon, only transitions among neighboring zones (i.e. previous and following one in the onedimensional domain) are taken into account. Similarly, one fracturon in zone $z$ can either enter the porous matrix and become a soluton in zone $z$ with transition rate $l_{f \rightarrow s, z}$ or travel to the nearby zones, $z+1$ or $z-1$, with transition rates $f_{f}(t)$ and $b_{f}(t)$. Finally, the contaminant might undergo a radioactive decay or a chemical reaction with rate $\lambda(t)$, upon which, for simplicity, we assume the particle disappears (the extension to follow chains of radionuclides and chemical species is conceptually trivial but adds complexity in the presentation of the model which are not meaningful for the scope of the presented work). Note that in general all the transition rates can depend on $t$, which implies the possibility of time-dependent interactions. Figure 1 summarizes the possible particle transitions within the one-dimensional migration domain.

To describe the space and time evolution of the system of particles, it is possible to write the following system of $2 N_{z}$ coupled differential equations (Forward Kolmogorov Equations) for the evolution of the expected values of the numbers of solutons $(S)$ and fracturons $(F)$ at time $t$ in zone [30]:

$$
\begin{aligned}
& \frac{\partial S\left(z^{\prime}, t \mid 1_{s, z}, 0\right)}{\partial t}=-\left[b_{s}(t)+f_{s}(t)+l_{s \rightarrow f, z^{\prime}}(t)+\lambda(t)\right] S\left(z^{\prime}, t \mid 1_{s, z}, 0\right)+b_{s}(t) S\left(z^{\prime}+1, t \mid 1_{s, z}, 0\right)+ \\
& +f_{s}(t) S\left(z^{\prime}-1 \mid 1_{s, z}, 0\right)+l_{f} \rightarrow s, z^{\prime}(t) F\left(z^{\prime}, t \mid 1_{s, z}, 0\right) \\
& \frac{\partial F\left(z^{\prime}, t \mid 1_{s, z}, 0\right)}{\partial t}=-\left[b_{f}(t)+f_{f}(t)+l_{f} \rightarrow s, z^{\prime}(t)+\lambda(t)\right] F\left(z^{\prime}, t \mid 1_{s, z}, 0\right)+b_{f}(t) F\left(z^{\prime}+1, t \mid 1_{s, z}, 0\right)+ \\
& +f_{f}(t) F\left(z^{\prime}-1 \mid 1_{s, z}, 0\right)+l_{s \rightarrow f, z^{\prime}}(t) S\left(z^{\prime}, t \mid 1_{s, z}, 0\right)
\end{aligned}
$$


with $z^{\prime}=1,2, \ldots, N_{z}$ and where we assume to have one soluton in zone $z$ at time $t=0\left(1_{s, z}\right)$. These equations appeal to our physical intuition as they represent the balance between the production and the destruction processes for both the solutons and the fracturons populations at $z^{\prime}$ in $d t$.

The forward and backward rates in equations (1.a,b) can be determined by analogy with the equivalent dual-permeability advection-dispersion equations [16][17], whereas the exchange rates can be identified on the basis of detailed physical analyses [16][31][32] (see Appendix A for more details).

When the transition rates are constant in time, the stochastic process of radionuclide migration modeled by the system of partial differential equations (1.a,b) represents a continuous-time homogeneous Markov process [33], whose solution is the probability of finding the $m^{\text {th }}$ contaminant particle in state $z^{\prime}$ at time $t$. Thus, in principle, analytic [33][34] or numerical [30] solutions of the system of coupled equations can be found. However, in realistic cases analytic solutions are difficult to obtain, if not impossible, whereas numerical schemes may, in some cases, be subject to restrictions in the choice of the time step in order to avoid numerical problems. Here we propose to resort to the Monte Carlo particle tracking scheme proposed in [16], which is directly derived from the underlying Markovian description of the stochastic evolution of the system of particles, whereby the stochastic migration of a large number $M$ of contaminant particles are simulated by repeatedly sampling their births from the release sources and their transitions across the medium compartments.

The random walk of the individual radionuclide particle is simulated either until it exits the domain to the $N_{z}+1$ compartment "environment", that is absorbing because from there the particle cannot come back into the migration domain, or until its lifetime crosses the time horizon $T$ of the analysis. With no loss of generality, the radioactive decay of the particles is taken into account at the end of the simulation by way of a convolution of the estimated concentrations and the decay probability density function (see the next Section). The time horizon $T$ is discretized in $N_{t}$ equally spaced time instants, with time step $\Delta t$. Two counters $\operatorname{Count}_{s}(n, k)$ and $\operatorname{Count}_{f}(n, k)$ are associated to each compartment $n=1,2, \ldots ., N_{z}+1$ and each discrete time $k=1,2, \ldots ., N_{t}$. During the simulation, a one is accumulated in the counters $\operatorname{Count}_{s}(n, k)$ and $\operatorname{Count}_{f}(n, k)$ if a soluton or a fracturon, respectively, resides, during the random walk, in compartment $n$ at time $k$. At the end of the $M$ simulated random walks of the contaminant particles, the values accumulated in the counters allow computing the estimates of the time-dependent probabilities of cell occupation by a soluton or a fracturon at the discrete times $k, \hat{P}_{n, s}(k)$ and $\hat{P}_{n, f}(k)$, respectively: 


$$
\begin{aligned}
\hat{P}_{n, s}(k) & =\frac{\text { Count }_{s}(n, k)}{M} \\
\hat{P}_{n, f}(k) & =\frac{\text { Count }_{f}(n, k)}{M}
\end{aligned}
$$

The total probability that a contaminant particle occupies cell $n$ at time $k$ is:

$$
\hat{P}_{n}(k) \cong \frac{\operatorname{Count}_{s}(n, k)+\operatorname{Count}_{f}(n, k)}{M}=\frac{\operatorname{Count}_{(}(n, k)}{M}
$$

Similarly, the point-wise approximation of the normalized release (mass flow) into the environment, $R_{e n v}(k)$, can be estimated as:

$$
\hat{R}_{\text {env }}(k) \cong \frac{\operatorname{Count}\left(N_{z}+1, k\right)}{M \cdot \Delta t}
$$

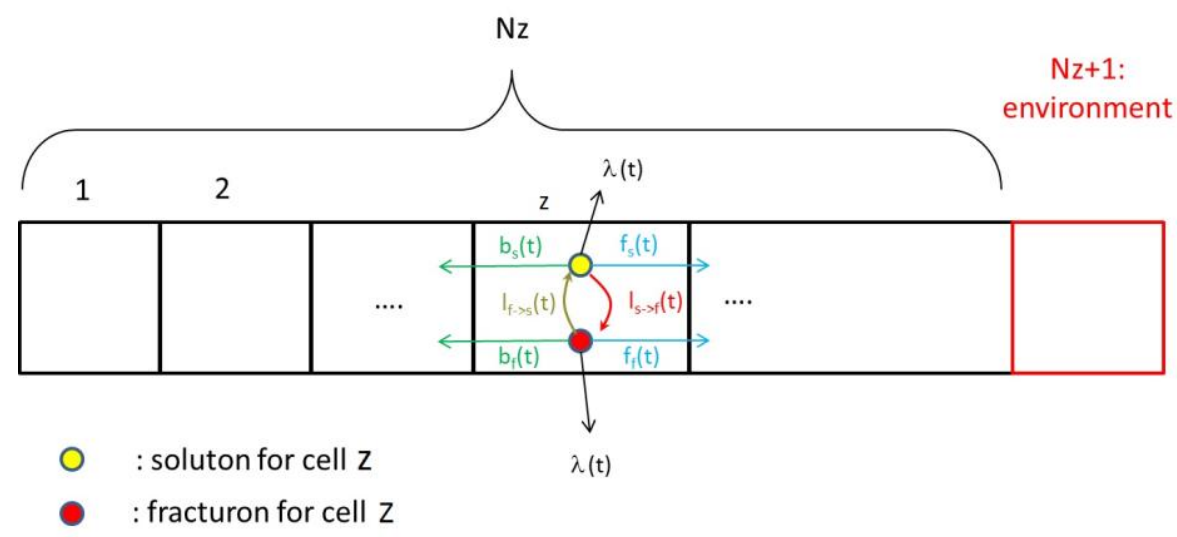

Figure 1: Possible particle transitions within the one-dimensional migration domain.

\section{RESULTS}

The dual-permeability, Monte Carlo simulation model is applied to a realistic case study of literature for the estimation of the expected dose due to the releases from a near surface repository. The near surface repository considered is a design concept studied by ENEA [29][35][36][37], and has similarities with the currently operative disposal facility of El Cabril, Spain [38]. As the radionuclides exit the repository floor, they migrate through the unsaturated zone, whose most important effect is that of retarding the infiltration towards the saturated zone [39]. Then, the 
radionuclide migration in the groundwater domain is described to occur within a highly fractured limestone layer exhibiting extensive karstic phenomena. The key hydrogeological features and hydraulic properties of the site, reported in Table 1, have been estimated by properly combining geophysical techniques and hydraulic testing through boreholes inspection at a repository candidate site [35].

\section{Table 1: Hydrologic data for groundwater migration of ${ }^{239} \mathrm{Pu}$ [35]}

\begin{tabular}{ll}
\hline Fracture porosity & $\phi_{f}=0.30$ \\
Matrix porosity & $\phi_{m}=0.30$ \\
Fracture tortuosity & $\tau_{f}=0.50$ \\
Matrix tortuosity & $\tau_{m}=0.50$ \\
Characteristic half width of matrix blocks & $a=0.3 \mathrm{~m}$ \\
Relative volume of the matrix system & $w_{m}=0.60$ \\
Relative volume of the fracture system & $w_{f}=0.40$ \\
Fracture hydraulic conductivity & $K_{f}=1 \cdot 10^{-3} \mathrm{~m} / \mathrm{s}$ \\
Matrix hydraulic conductivity & $K_{m}=1 \cdot 10^{-6} \mathrm{~m} / \mathrm{s}$ \\
Average hydraulic gradient & $\Delta h / \Delta z=0.003$ \\
Molecular diffusion coefficient & $D_{m o l}=1 \cdot 10^{-9} \mathrm{~m}^{2} / \mathrm{s}$ \\
Fracture transverse and longitudinal dispersivity & $\alpha_{T, f}=0.0 \mathrm{~m}, \alpha_{L, f}=10.0 \mathrm{~m}$ \\
Matrix transverse and longitudinal dispersivity & $\alpha_{T, m}=0.0 \mathrm{~m}, \alpha_{L, m}=5.0 \mathrm{~m}$ \\
Fracture-matrix dispersivity & $\alpha_{f-m}=0.0 \mathrm{~m}$ \\
Plutonium retardation coefficient & $R=4167$ \\
Plutonium radioactive decay & $\lambda_{239}=0.28761 \cdot 10^{-4} y^{-1}$ \\
Geometric fracture parameter & $\beta=3(\mathrm{rectangular} \mathrm{slab)}$
\end{tabular}

The dual-permeability, Monte Carlo model of Section 2 is employed to simulate the transport of the radionuclide ${ }^{239} \mathrm{Pu}$ within the groundwater body described above; this particular radionuclide has been chosen because it represents a long-term threat to the environment for its radioactivity $\left(T_{1 / 2}=2.411 \cdot 10^{4} \mathrm{y}\right)$ and toxicity. To promote model simplicity for repeated use in PA probabilistic analyses, a one-dimensional model is considered. To simulate the transport, $M=10^{5}$ fracturon particles are injected, according to the release rate computed in [29], in the first upstream 
compartment of a dual permeability, one-dimensional array of $N_{z}=50$ compartments of equal width $\Delta z=2 \mathrm{~m}$, representing both the fracture and the porous matrix, and simulated over a time horizon of $T=10000 y$, divided into $N_{t}=500$ equal time steps of constant width $\Delta t=20 y$. The migration of ${ }^{239} \mathrm{Pu}$ in the groundwater is assumed to occur under linear isothermal conditions. The backward and forward transition rates for both the solutons and the fracturons, $b_{s}, f_{s}$ and $b_{f}, f_{f}$, respectively, and the exchange rates $l_{s \rightarrow f, z}$ and $l_{f} \rightarrow s, z$ are computed by resorting to equations (A5.a,b) and (A7.a,b), with the parameter values of Table 1 [35] and $D=\alpha_{L} v+\phi D_{m o l} \tau$. The geometric parameters $a$ and $\beta$ are set to the values of Table 1 on the basis of engineering common sense, due to the lack of a more detailed characterization of the fracture system but with no loss of generality.

At the end of the $M$ simulated random walks, the values accumulated in the counters (see Section 2) allow estimating the time-dependent probabilities of compartment occupation $\hat{P}_{n}(k)$, which can in turn be employed to estimate the ${ }^{239} \mathrm{Pu}$ concentration and therefore the expected dose to the population. For illustration purposes, let us consider a groundwater compartment of depth $\Delta y=1 \mathrm{~m}$, width $\Delta x=250 \mathrm{~m}$ and length equal to the spatial discretization step $\Delta z=2 \mathrm{~m}$ [39]. Thus, the volume of the groundwater domain compartment is $\Delta V=\Delta x \cdot \Delta y \cdot \Delta z=500.00 \mathrm{~m}^{3}$. By neglecting for simplicity the ${ }^{239} \mathrm{Pu}$ radionuclides that are generated by the decay chains of other radioactive elements contained in the repository, the ${ }^{239} P u$ time dependent concentration $C(n, k)$ $\left[\mathrm{Bq} / \mathrm{m}^{3}\right]$ in a generic groundwater domain compartment $n$ at time $k$ can be estimated as:

$$
C(n, k) \cong \hat{P}_{n}(k) \cdot \frac{A_{239} P u}{\Delta x \cdot \Delta y \cdot \Delta z}=\frac{\operatorname{Count}_{s}(n, k)+\operatorname{Count}_{f}(n, k)}{M \cdot \Delta V} \cdot A_{239 P u}(k)
$$

where $A_{239}(k)[\mathrm{Bq}]$ is the total activity of ${ }^{239} \mathrm{Pu}$ and is computed as

$$
A_{239 P u}(k)=A_{0} e^{-\lambda_{239} p_{u} k}
$$

where $\lambda_{239}=2.8761 \cdot 10^{-5} y^{-1}$ is the decay rate for ${ }^{239} \mathrm{Pu}$ and $A_{0}=1.6 \cdot 10^{10} \mathrm{~Bq}$ is the total ${ }^{239} \mathrm{Pu}$ inventory at a reference time $t=0[40]$. 
On the basis of the estimated ${ }^{239} \mathrm{Pu}$ concentrations in groundwater, the final dose intensity to humans $D_{w}[S v / y]$ by ingestion of drinking water can be estimated as [35]:

$$
D_{w}(n, k)=C_{\text {acquifer }}(n, k) I N G_{w} D C_{\text {ing }}
$$

where $I N G_{w}=7.3 \cdot 10^{-1} \mathrm{~m}^{3} / y$ is the average quantity of drinking water consumed per year and $D C_{\text {ing }}=15.7 \cdot 10^{-9} \mathrm{~Sv} / \mathrm{Bq}$ is the ${ }^{239} \mathrm{Pu}$ dose conversion factor for ingestion [35].

Figure 2 shows the estimate of the expected dose at a groundwater compartment located 100 meters downstream of the source compartment (dotted line). For decision making purposes, the estimated ${ }^{239} \mathrm{Pu}$ dose intensities must be compared to the limit prescribed by the Italian law for the dose to the population, i.e., a total of $1 \mathrm{mSv} / \mathrm{y}$ above the level of $2.4 \mathrm{mSv} / \mathrm{y}$ due to the background natural radioactivity [35][41]. The maximum dose intensities from ${ }^{239} \mathrm{Pu}$ estimated, about $0.15 \mathrm{mSv} / \mathrm{y}$, are below the above limit indirectly confirming the reasonableness of the assumptions made. The long "tail" of the dose starting from approximately 4000 years is due to the matrixfracture exchange phenomenon. Figure 2 shows in fact the expected dose (dotted line) compared to the curves computed taking into account only the contaminant particles which have undergone no exchange transitions (solid line) and those which have undergone an exchange transition at least once (dashed line). As expected, the shape and the time of the dose peak is due to those fracturons which exit the domain, without any interaction with the matrix, whereas the long tail is made up of the particles delayed by the interaction with the matrix.

The effects of the matrix-fracture exchange phenomenon is further investigated by running two additional MC simulations with different exchange rates. Figure 3 shows the expected doses with $l_{f \rightarrow s, z}=l_{s \rightarrow f, z}=0$ (solid line) and $l_{f \rightarrow s, z}=3.8 \cdot 10^{-2} y^{-1}$ and $l_{s \rightarrow f, z}=2.5 \cdot 10^{-2} y^{-1}$, i.e. two orders of magnitude larger than in the first case, (dashed line). The first case is equivalent to modeling the radionuclide migration in a single-permeability domain with hydrologic properties of the sole fractures. The expected dose shows a larger peak and a shorter tail, due to the fact that the fracturons are not delayed by the exchange process with the porous matrix; at the same time, the peak time remains the same, because in the previous case depended only on the fraction of fracturons which does not interact with the matrix before exiting the domain. In the second case, the exchange rates are increased to values which imply that all the fracturons become solutons at least once before exiting (as verified by the authors, but not shown here for brevity's sake), thus causing a shift of the peak towards larger time and a broadening of the curve due to larger dispersion. 


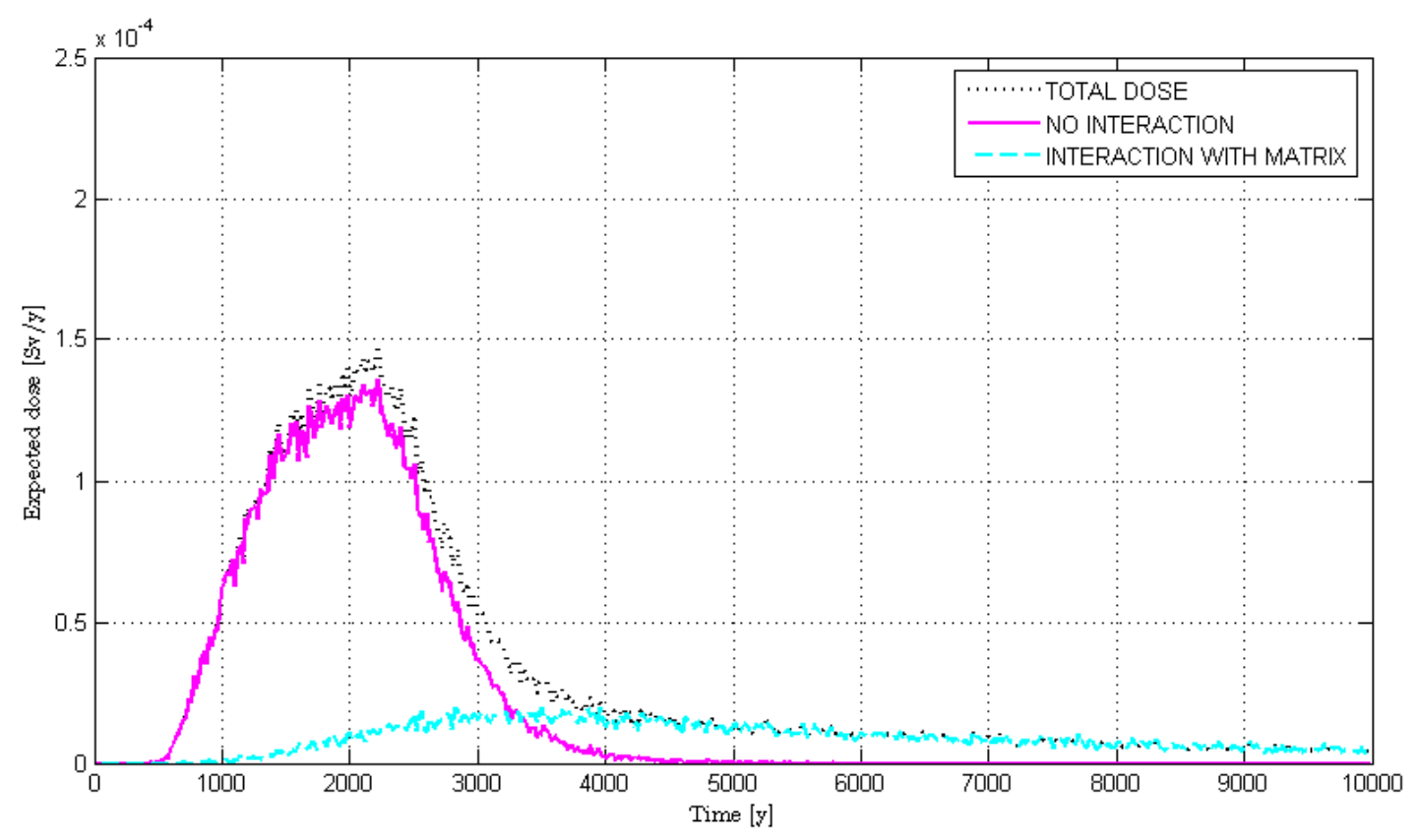

Figure 2: Comparison between total dose (dotted line), contribution of the contaminant particles which exit the domain without interacting (solid line) and those which are delayed by the exchange with the matrix (dashed line).

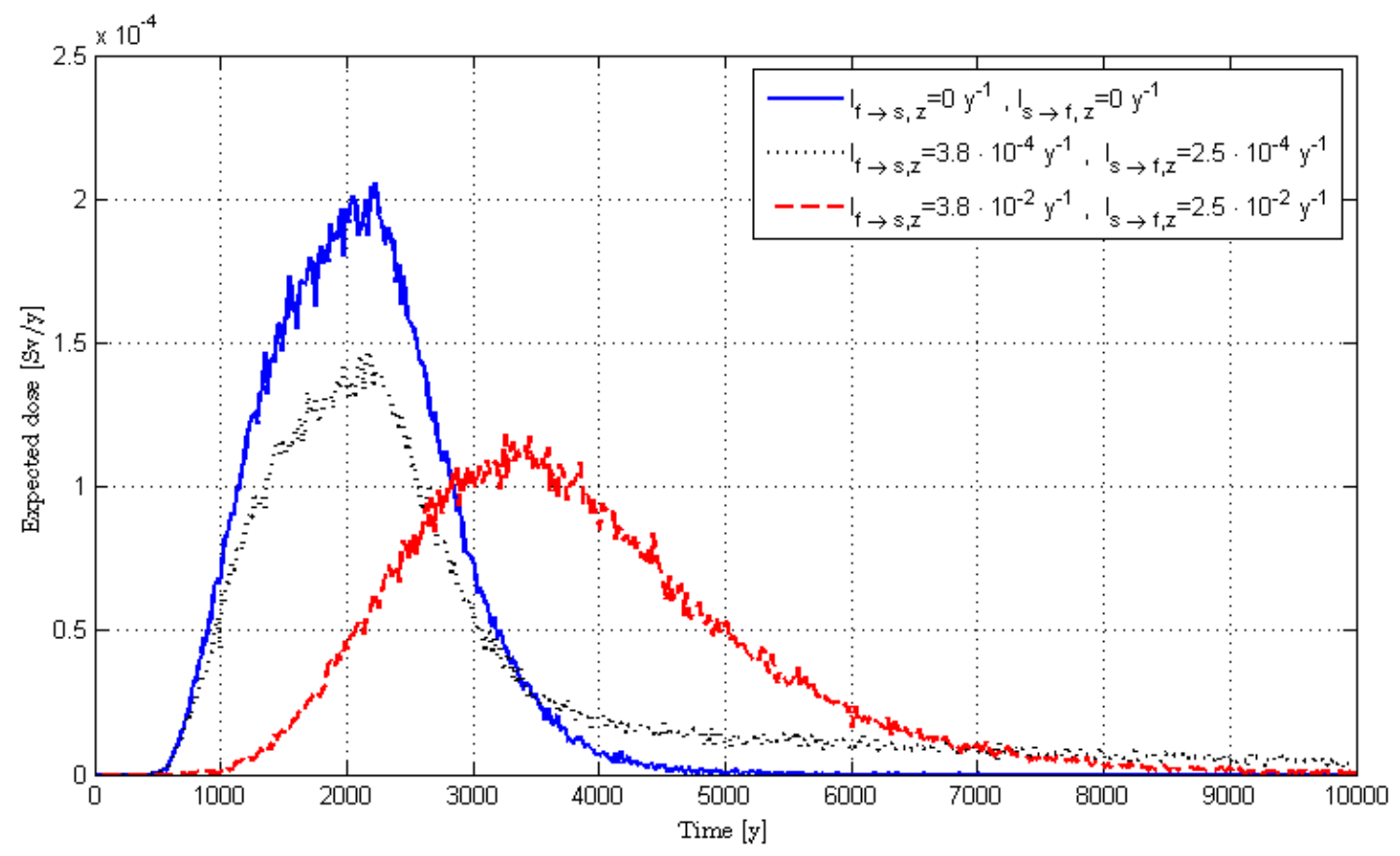

Figure 3: Comparison between the expected doses in the dual-permeability base case (dotted line), the singlepermeability case (solid line) an dual-permeability case with increased exchange rates (dashed line). 
As mentioned in Section 2, the flexibility of the adopted model allows taking into account more realistic features of the domain and phenomena typically involved in the migration process.

This capability is first exploited on an extension of the case study previously described, which involves long term modifications of the groundwater gradient due to climate changes (e.g. rainfalls, glaciation, etc.) [42]. The process is modeled as a continuous increase in time of the groundwater fracture pore velocity $v_{f}$ : for simplicity, but with no loss of generality, it is assumed that the change can be described by the following power law

$$
v_{f}=\alpha \beta v_{f, 0} t^{\alpha-1}
$$

where $\alpha$ and $\beta$ are model parameters and $v_{f, 0}$ is the initial value of the pore velocity in fractures, assumed equal to the value adopted in the previous case study. Thus, the forward and backward transition rates of the fracturons, $f_{f}$ and $b_{f}$, become time-dependent functions (see equations (A5.a,b) ). As in the previous case study, $M=10^{5}$ fracturon particles are injected, according to the release rate computed in [29], in the first upstream compartment of the same dual permeability, onedimensional domain and simulated over a time horizon of $T=10000 y$, discretized into $N_{t}=500$ time steps with constant width $\Delta t=20 y$. The exchange rates are set equal to $l_{s \rightarrow f, z}=2.5 \cdot 10^{-2} y^{-1}$ and $l_{f \rightarrow s, z}=3.8 \cdot 10^{-2} y^{-1}$ and the parameters $\alpha$ and $\beta$ to 1.15 and 0.87 , respectively, which amounts to gradually increase the pore velocity value to a maximum of approximately $v_{f} \cong 4 \cdot v_{f, 0}$ at $T=10000 y$. All the remaining physical and simulation parameters are equal to those used in the previous case study. Figure 4 shows the estimate of the expected dose at a compartment 100 meters downstream of the source (dotted line); the comparison with the curve previously obtained with constant forward and backward transition rates, $f_{f}=2.08 \cdot 10^{-1} y^{-1}$ and $b_{f}=1.70 \cdot 10^{-1} y^{-1}$, and same exchange rates (dashed line), shows that the peak time shifts towards earlier times and the dispersion decreases. These effects were to be expected since, on average, the pore velocity is larger and, consequently, the probability of a fracturon being absorbed by the matrix sensibly decreases, thus leading to results similar to those obtained with a singlepermeability model (Figure 2, solid line).

The same case study is then extended to consider that the aperture of the fractures may stochastically vary in time due to the mechanical stresses induced by earthquakes hitting the migration domain [27]. The apertures of fractures may change due to normal stress-induced closures or openings and to shear stress-induced dilations; thus, the permeability of fracture rock 
masses is stress-dependent [24][26]. This indirect hydro-mechanical coupling is particularly important in fractured rock masses, since stress-induced changes in permeability can be large (several orders of magnitude) and irreversible under, in general, perturbations resulting from various natural and human activities [24]. For simplicity, but with no loss of generality, we now assume that i) the times at which the earthquakes occurr is distributed according to an exponential distribution with parameter $v=8.3 \cdot 10^{-3} \mathrm{y}^{-1}$ [43], which is the average return rate of magnitude $4 / 5$ earthquakes of the site proposed in [35] and ii) each earthquake occurrence induces a fracture permeability change which, on average, conservatively increases the pore velocity by a $10 \%$ factor, starting from the initial value $v_{f, 0}$. As in the previous example, this entails a modification of the forward and backward transition rates of the fracturons according to equations (A5.a,b) and to $D=\alpha_{L} v+\phi D_{m o l} \tau$. Figure 4 (solid line) shows the results of a simulation with the forward and backward transition rates dependent on earthquake stress-induced effects and all remaining parameters identical to those of the previous example. During the simulation, a different earthquake sequence is generated over the time horizon by sampling the occurrence times from the exponential distribution in correspondence of every particle random walk. With respect to the base case illustrated before (dashed line), the peak time occurs earlier and the dispersion of the curve decreases, although to a minor extent than in the previous example: in fact, as verified by the authors, the pore velocity is on average smaller.

In conclusion, with the data used in both cases of time-dependent migration properties, the compliance of the expected doses with the regulatory limits introduced above is verified. 


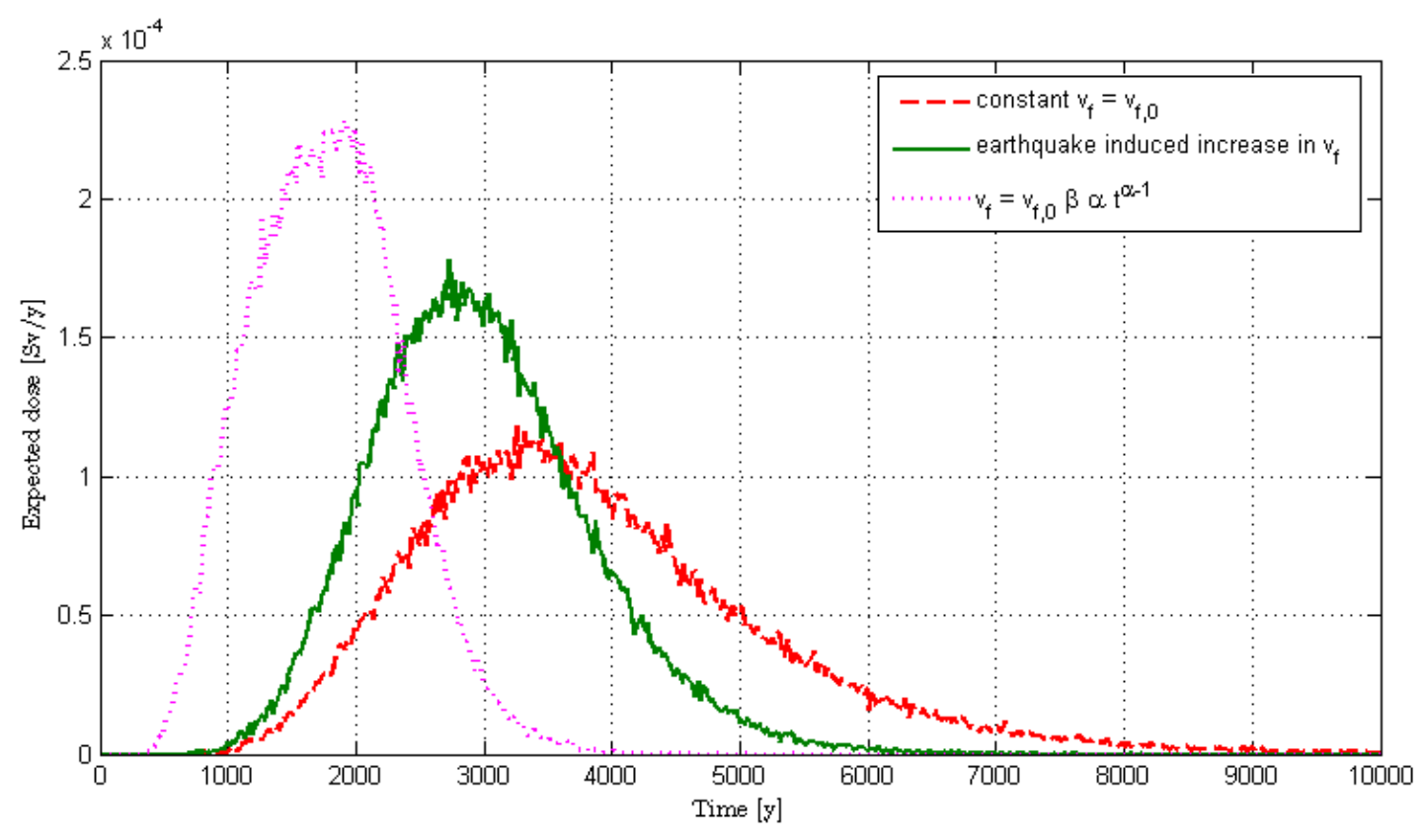

Figure 4: Dose curve $100 \mathrm{~m}$ from the source: comparison between the time-constant rates and the case with variable rates.

\section{CONCLUSIONS}

The performance assessment of radioactive waste repositories relies on predictive models for the quantitative analysis of the consequences of radionuclide releases, which are then transported back to the biosphere along natural pathways. A particular concern is the migration of radionuclides in the preferential pathways offered by fractures in ground formations traversed by a groundwater flow.

In this paper, a dual-permeability, Monte Carlo simulation scheme based on the KolmogorovDmitriev theory of stochastic branching processes for modeling radionuclide migration in a fractured medium has been embedded within a dose assessment problem of a realistic radioactive waste repository. The method has allowed to capture the effects of the fracture-matrix interactions on the migration process, which may affect the overall safety considerations in terms of the peak dose and the persistence of the dose tails.

The flexibility offered by the Monte Carlo simulation approach has been exploited to capture the effects of time-dependent changes in the hydraulic properties of the fractures on the predicted dose. The results have demonstrated that the method allows assessing how robust is the safety performance with respect to the occurrence of long-term gradual climate changes or discrete catastrophic events. 


\section{APPENDIX A}

In dual permeability and dual porosity models, the transport phenomenon is assumed to occur in two domains: the porous matrix and the fracture system. In particular, dual porosity models divide the medium in a mobile and an immobile region, assuming that flow occurs only in the larger permeability zone, whereas dual-permeability models assume the flow occurs in both subsystems.

The model describes the heterogeneous porous media as a system made up of two different coupled subsystems and therefore the properties of the bulk medium depend on those of the different porous subsystems. Thus, following the notation of [31][32], the transport of solute in this system can be described by two coupled advection-reaction-dispersion equations, where the subscript $m$ refers to the matrix subsystem and $f$ to the fractures:

$$
\begin{gathered}
\frac{\partial\left(\theta_{f} R_{f} c_{f}\right)}{\partial t}=\nabla \cdot\left(\theta_{f} \mathbf{D}_{\mathbf{f}} \nabla c_{f}\right)-\nabla \cdot\left(\mathbf{q}_{\mathbf{f}} c_{f}\right)-\theta_{f} \lambda_{f} c_{f}-\frac{\Gamma_{s}}{w_{f}} \\
\frac{\partial\left(\theta_{m} R_{m} c_{m}\right)}{\partial t}=\nabla \cdot\left(\theta_{m} \mathbf{D}_{\mathbf{m}} \nabla c_{m}\right)-\nabla \cdot\left(\mathbf{q}_{\mathbf{m}} c_{m}\right)-\theta_{m} \lambda_{m} c_{m}+\frac{\Gamma_{s}}{w_{m}}
\end{gathered}
$$

where $\mathbf{D}$ is the dispersion tensor, $\lambda$ is the decay or chemical reaction term, $R$ is the retardation coefficient, $w$ is the relative volume of the pore system $\left(w_{m}=1-w_{f}\right), \theta$ is the water content, $\mathbf{q}$ is the Darcy's velocity, $c$ is the solute concentration and $\Gamma_{s}$ is the exchange term between the porous matrix and the fracture system. According to [31][32], assuming that only the diffusive contribution to the exchange process is taken into account and that the flow field is at steady-state, the exchange term $\Gamma_{s}$ can be expressed as:

$$
\Gamma_{s}=\alpha_{s} w_{m} \theta_{m}\left(c_{f}-c_{m}\right)
$$

The coefficient $\alpha_{s}$ in equation (3) is [31][32]:

$$
\alpha_{s}=\frac{\beta}{a^{2}} D_{a}
$$

where $D_{a}$ is the effective diffusion coefficient at the interface, $\beta$ is a semi-empirical geometric factor and $a$ is the characteristic half-width of the porous matrix. The terms in equations (2.a,b) are 
all referred to the relative volume of the corresponding subsystem, with the exception of $\Gamma_{s}$, which is defined as the mass of solute per unit volume of bulk soil per unit time [31]. For the general form of equations (A1.a,b) and (A2) the interested reader should refer to [31][32].

Then under the hypotheses of: $i)$ one-dimensional domain, ii) no retardation $(R=1)$ and $i i i)$ constant water contents $\left(\theta_{m}\right.$ and $\left.\theta_{f}\right)$, constant pore velocities $v_{m}=\frac{q_{m}}{\phi_{m}} v_{f}=\frac{q_{f}}{\phi_{f}}\left(\right.$ where $\phi_{m}$ and $\phi_{f}$ are the porosities of the matrix and the fractures system, respectively), constant dispersion coefficients $D_{m}$ and $D_{f}$ and constant reaction rates $\lambda_{m}=\lambda_{f}=\lambda$, it is possible to write equations (2.a,b) in terms of the concentrations referred to the total bulk volume $\left(C_{f}=\theta_{f} w_{f} c_{f}\right.$ and $\left.C_{m}=\theta_{m} w_{m} c_{m}\right)$ and, subsequently, discretize them in space by a centered Euler method:

$$
\begin{aligned}
\frac{\partial C_{f}}{\partial t}= & -v_{f} \frac{\left(C_{f}(z+1, t)-C_{f}(z-1, t)\right)}{\Delta z}+D_{f} \frac{\left(C_{f}(z+1, t)+C_{f}(z-1, t)-2 C_{f}(z, t)\right)}{\Delta z^{2}}- \\
& -\alpha_{s} \frac{\theta_{m} w_{m}}{\theta_{f} w_{f}} C_{f}(z, t)+\alpha_{s} C_{m}(z, t)-\lambda C_{f}(z, t) \\
\frac{\partial C_{m}}{\partial t}= & -v_{m} \frac{\left(C_{m}(z+1, t)-C_{m}(z-1, t)\right)}{\Delta z}+D_{m} \frac{\left(C_{m}(z+1, t)+C_{m}(z-1, t)-2 C_{m}(z, t)\right)}{\Delta z^{2}}+ \\
& +\alpha_{s} \frac{\theta_{m} w_{m}}{\theta_{f} w_{f}} C_{f}(z, t)-\alpha_{s} C_{m}(z, t)-\lambda C_{m}(z, t)
\end{aligned}
$$

These equations are formally identical to (1.a,b); thus, by comparing the two systems of equations it is possible to write the following relationships for the forward and backward rates of the solutons and the fracturons:

$$
\begin{gathered}
f_{s}=\frac{D_{m}}{\Delta z^{2}}+\frac{v_{m}}{2 \Delta z} ; \quad f_{f}=\frac{D_{f}}{\Delta z^{2}}+\frac{v_{f}}{2 \Delta z} \\
b_{s}=\frac{D_{m}}{\Delta z^{2}}-\frac{v_{m}}{2 \Delta z} ; \quad b_{f}=\frac{D_{f}}{\Delta z^{2}}-\frac{v_{f}}{2 \Delta z}
\end{gathered}
$$

Note that, since the backward transition rates $b_{s}$ and $b_{f}$ cannot be negative, equations (A5.b) require an upper limit for the spatial discretization cell:

$$
\Delta z<\min \left[\frac{2 D_{m}}{v_{m}} ; \frac{2 D_{f}}{v_{f}}\right]
$$


Analogously to the forward and backward rates, the exchange rates can be expressed as:

$$
\begin{gathered}
l_{f \rightarrow s, z}=\alpha_{s} \frac{\theta_{m} w_{m}}{\theta_{j} w_{f}} \\
l_{s \rightarrow f, z}=\alpha_{s}
\end{gathered}
$$

The difference in the exchange rates depends mainly upon the different relative volume of each system: the larger the volume of the subsystem is, the less probable the transition to the other subsystem is.

\section{REFERENCES}

[1] Painter, S. and Cvetkovic, V., Upscaling discrete fracture network simulations: An alternative to continuum models. Water Resour. Res., 41, W02002, doi:10.1029/2004WR003682, 2005.

[2] Ramsey JL, Blaine R, Garner JW, Helton JC, Johnson JD, Smith LN, Wallace M. Radionuclide and Colloid Transport in the Culebra Dolomite and Associated Complementary Cumulative Distribution Functions in the 1996 Performance Assessment for the Waste Isolation Pilot Plant. Reliability Engineering and System Safety 2000; 69(1-3):397-420.

[3] Golubev, V.S. and Garibyants, A.A., Heterogeneous Processes of Geochemical Migration, New York, Consultants Bureau, 1971.

[4] Vaughn P, Bean JE, Helton JC, Lord ME, MacKinnon RJ, Schreiber JD. Representation of Two-Phase Flow in the Vicinity of the Repository in the 1996 Performance Assessment for the Waste Isolation Pilot Plant. Reliability Engineering and System Safety 2000; 69(1-3):205-226.

[5] Helton JC, Bean JE, Economy K, Garner JW, MacKinnon RJ, Miller J, Schreiber JD, Vaughn P. Uncertainty and Sensitivity Analysis for Two-Phase Flow in the Vicinity of the Repository in the 1996 Performance Assessment for the Waste Isolation Pilot Plant: Undisturbed Conditions. Reliability Engineering and System Safety 2000; 69(1-3):227-261.

[6] SNL (Sandia National Laboratories). UZ Flow Models and Submodels. MDL-NBS-HS-000006 REV 03 AD 01. Las Vegas, NV: U.S. Department of Energy Office of Civilian Radioactive Waste Management; 2007. 
[7] Zyvoloski AG. FEHM: A Control Volume Finite Element Code for Simulating Subsurface Multi-Phase Multi-Fluid Heat and Mass Transfer. LA-UR-07-3359. Los Alamos, NM: Los Alamos National Laboratory; 2007.

[8] SNL (Sandia National Laboratories). Particle Tracking Model and Abstraction of Transport Processes. MDL-NBS-HS-000020 REV 02 AD 02. Las Vegas, NV: U.S. Department of Energy Office of Civilian Radioactive Waste Management; 2008.

[9] SNL (Sandia National Laboratories). Site-Scale Saturated Zone Transport. MDL-NBS-HS000010 REV 03 AD 01 ACN001. Las Vegas, NV: U.S. Department of Energy Office of Civilian Radioactive Waste Management; 2008.

[10] SNL (Sandia National Laboratories). Saturated Zone Flow and Transport Model Abstraction. MDL-NBS-HS-000021 REV 03 AD 02. Las Vegas, NV: U.S. Department of Energy Office of Civilian Radioactive Waste Management; 2008.

[11] Mattie PD, Hadgu T, Lester B, Smith A, Wasiolek M, Zwahlen E. Yucca Mountain 2008 Performance Assessment: Modeling the Natural System. In. Proceedings of the 2008 International High-Level Radioactive Waste Management Conference, September 7-11, 2008: La Grange Park, IL: American Nuclear Society, 2008:550-558”.

[12] Tang, D.H, Frind, E.O. and Sudicky E.A., Contaminant Transport in Fractured Porous Media: Analytical Solution for a Single Fracture, Water Resour. Res., 17(3), 555-564, 1981.

[13] Neretnieks, I., Diffusion in the rock matrix: an important factor in radionuclide retardation?, $J$. Geophys. Res., 85, 4379-4397, 1980.

[14] Grisak, G. E., and Pickens, J. F. , Solute transport through fractured media: 1. The effect of matrix diffusion, Water Resour. Res., 16(4), 719-730, doi:10.1029/WR016i004p00719, 1980.

[15] Sudicky, E.A., Gillham, R.W. and Frind, E.O., Experimental Investigation of Solute Transport in Stratified Porous Media: 1 The Nonreactive Case, Water Resour. Res., 24(7), 1035-1041, 1985.

[16] Cadini F., Bertoli I., De Sanctis J. and Zio E., A novel particle tracking scheme for modeling contaminant transport in a dual-continua fractured medium, Water Resources Research, in press, doi:10.1029/2011WR011694.

[17] Ferrara A., Maseguerra M., Zio E., A comparison between the advection-dispersion and the Komogorov-Dmitriev model for groundwater contaminant transport. Ann. Nucl. Energy, 26(12), 1083-1096, 1999.

[18] Cadini, F., De Sanctis, J. and Zio, E., Challenges in Monte Carlo simulation of non-linear radioactive contaminant release processes. In Proceedings of the 13th International High-Level Radioactive Waste Management Conference, IHLRWMC 2011, 2011. 
[19] Langer, M., Principles of geomechanical safety assessment for radioactive waste disposal in salt structures. Engineering geology, 52(3-4):257 269, 1999.

[20] Manepally, C. and Randall, F., Evaluation of coupled thermal-hydrological-mechanicalchemical processes for deep disposal in different geologic media. In Proceedings of the 13th International High-Level Radioactive Waste Management Conference, IHLRWMC 2011, 2011.

[21] Lowell R.P., Van Cappellen, P. and Germanovich, L.N., Silica Precipitation in Fractures and the Evolution of Permeability in Hydrothermal Upflow Zones, Science, 260 (5105), 192-194, 1993. [22] Fetter, C.W., Applied Hydrogeology, 4ed. Prentice Hall Upper Saddle, River, N.J., 2001.

[23] Bai, M. and Elsworth, D., Modeling of Subsidence and Stress- Dependent Hydrulic Conductivity for Intact and Fractured Porous Media. Rock Mech. And Rock Eng., 27(4):209-234, 1994.

[24] Min, K.B., Rutqvist, J. Tsang, C.F. and Jing, L., Stress-dependent permeability of fractured rock masses: a numerical study. Int. J. Rock Mech. Min. Sci, 41G:1191-1210, 2004

[25] Mukhopadhyay, S., Sonnenthal, E.L. and Spycher, N., Modeling coupled thermalhydrological-chemical processes in the unsaturated fractured rock of Yucca Mountain, Nevada: Heterogeneity and seepage, Physics and Chemistry of the Earth, 31, 626-633, 2006.

[26] Rutqvist, J. and Tsang C.F., Analysis of thermal-hydrologic-mechanical behavior near an emplacement drift at Yucca Mountain, J. Contaminant Hydrology, 62-63, 637-652, 2003.

[27] Elkhoury J.E., Brodsky E.E. and Agnew D.C., Seismic waves increase permeability, Nature, 441, 1135-1138, doi:10.1038/nature04798, 2006

[28] Helton JC, Bean JE, Economy K, Garner JW, MacKinnon RJ, Miller J, Schreiber JD, Vaughn P. Uncertainty and Sensitivity Analysis for Two-Phase Flow in the Vicinity of the Repository in the 1996 Performance Assessment for the Waste Isolation Pilot Plant: Disturbed Conditions. Reliability Engineering and System Safety 2000; 69(1-3):263-304.

[29] Cadini, F., De Sanctis, J., Girotti, T., Zio, E., Luce, A. and Taglioni, A., Monte Carlo estimation of radionuclide release at a repository scale. Ann. Nucl. Energy, 37:861-866, 2010.

[30] Marseguerra M. and Zio E., Modelling the Transport of Contaminants in Groundwater as a Branching Stochastic Process, Ann. Nucl. Energy, 24(8), 325-6444, 1997.

[31] Gerke H.H. and van Genuchten M.T., A Dual-Porosity Model for Simulating the Preferential Movement of Water and Soluted in Structured Porous Media, Water Resour. Res., 29(2), 305-319, 1993.

[32] Gerke H.H. and van Genuchten M.T., Evaluation of a First-Order Water Transfer Term for Variably Saturated Dual-Porosity Flow Models, Water Resour. Res., 29(4), 1225-1238, 1993. 
[33] Papoulis, A. and Pillai, S.U., Probability, random variables, and stochastic processes. McGrawHill, 2002

[34] Zio, E., Computational methods for reliability and risk analysis, volume 14. World Scienti c Pub Co Inc, 2009.

[35] Internal Technical report, ENEA, 1997.

[36] Marseguerra, M., et al., 2001. Sviluppo di un Modello Stocastico e sua Implementazione in un Codice Monte Carlo. Internal Report, Politecnico di Milano (in Italian).

[37] Cadini, F., De Sanctis, J., Girotti, T., Zio, E., Luce, A., Taglioni, A., Monte Carlo-based assessment of the safety performance of a radioactive waste repository, Reliability Engineering and System Safety, 95, 859-865, 2010.

[38] Zuloaga, P., Andrade, C. and Saaltink, M.W. Long term water scenario in low-level waste disposal vaults, with particular regard to concrete structures in el cabril, cordoba, spain. In Journal de Physique IV (Proceedings), volume 136, pages 49-59, 2006.

[39] Cadini, F., De Sanctis, J., Girotti, T., Zio, E., Luce, A., and Taglioni, A., Monte Carlo-based assessment of the safety performance of a radioactive waste repository. Rel. Eng. And Syst. Safety, 95,859-865, 2010.

[40] ENEA. Inventario nazionale dei rifiuti radioattivi. Task force per il sito nazionale di deposito dei materiali radioattivi, 3 a edizione, 2000.

[41] ENEA. Internal report, Agenzia nazionale per le nuove tecnologie, l'energia e lo sviluppo economico sostenibile, 1997.

[42] Garisto, F.; Walsh, R.; Wojciechowski, L.; Avis, J.; Chshyolkova, T.; Gierszewski, P.; Gobien, M.; Kitson, C.; Melnyk, T. and Miller J., Safety assessment of a glaciation scenario. In Proceedings of the 13th International High-Level Radioactive Waste Management Conference, IHLRWMC 2011, 2011.

[43] Stucchi, M., Camassi, R., Rovida, A., Locati, M., Ercolani, E., Meletti, C., Migliavacca, P., Bernardini, F., Azzaro, R. et al., Dbmi04, il database delle osservazioni macrosismiche dei terremoti italiani utilizzate per la compilazione del catalogo parametrico cpti04. Quaderni di Geofisica, 2007 (In Italian). 